\title{
THE INTERIOR POINTS OF THE PRODUCT OF TWO SUBSETS OF A LOCALLY COMPACT GROUP
}

\author{
ANATOLE BECK, ${ }^{1}$ H. H. CORSON,${ }^{2}$ AND A. B. SIMON ${ }^{2}$
}

1. Introduction. Recently, B. J. Pettis conjectured that under certain hypotheses (see Theorem 1 below) the product of two subsets of a locally compact topological group should have a nonvoid interior. The purpose of this paper is to prove this statement. We then use this result to prove, among other things, a theorem of Simon's [1], which he established by a different method.

The conjecture is an analog of earlier work by E. J. McShane [2] and B. J. Pettis $[3 ; 4]$, namely:

Theorem (McShane). Let $G$ be a topological group and let $A, B \subset G$ be sets of second category with one of them satisfying the condition of Baire. Then the interior of $A B$ is not empty.

Our theorem reads:

TheOREM 1. Let $G$ be a locally compact topological group with completed Haar measure $\mu$ and outer measure $\mu^{*}$. Let $A, B \subset G$ be sets such that $\mu(A)>0$ and $\mu^{*}(B)>0$. Then the interior of $B A$ (also $A B$ ) is nonvacuous.

In order to show this, we prove the following lemma:

Lemma 1.1. Let $G$ be a locally compact group with completed Haar measure $\mu$ and outer measure $\mu^{*}$. Let $A, B \subset G$ be sets such that $A$ has finite measure and $B$ is contained in a set of finite measure. If the function $f$ is defined by $f(x)=\mu^{*}(x A \cap B)$, then $f$ is continuous and $\int_{G} f(x) d \mu(x)$ $=\mu\left(A^{-1}\right) \mu^{*}(B)$.

As an application of Theorem 1, we prove:

TheOREM 3 (Simon [1]). In a compact group, every subsemigroup which contains a set of positive measure is an open and closed subgroup; and therefore is itself measurable.

2. Proof of Theorem 1. We first prove Lemma 1.1 which was stated in the introduction.

Received by the editors May 27, 1957.

${ }^{1}$ ONR Research Associate.

2 Supported in part by the National Science Foundation. 
Proof of Lemma 1.1. To show $f$ is continuous, let $\epsilon>0$ be given. We can find a neighborhood $U$ of the identity with the property that $\mu(x A \Delta x u A)=\mu(A \Delta u A)<\epsilon$ for all $u \in U$ and $x \in G$. Now $(x A \cap B)$ $\cdot \Delta(x u A \cap B)=(x A \Delta x u A) \cap B$; hence $\mu^{*}((x A \cap B) \Delta(x u A \cap B))<\epsilon$, if $u \in U$. Since $x A$ and $x u A$ are measurable, we have

$$
\mu^{*}(x A \cap B)=\mu^{*}((x A \cap B) \cap x u A)+\mu^{*}((x A \cap B) \backslash x u A),
$$

and

$$
\mu^{*}(x u A \cap B)=\mu^{*}((x u A \cap B) \cap x A)+\mu^{*}((x u A \cap B) \backslash x A) .
$$

Applying these formulas to the definition of $f$, it follows that $|f(x)-f(x u)|=\left|\mu^{*}((x A \cap B) \backslash x u A)-\mu^{*}((x u A \cap B) \backslash x A)\right|$ $\leqq \mu^{*}((x A \cap B) \Delta(x u A \cap B))<\epsilon$ for all $u \in U$ and $x \in G$; and $f$ is continuous.

Now let $\mu\left(A^{-1}\right)=\alpha<\infty$ and $\mu^{*}(B)=\beta<\infty$, and let the sequence of sets $\left\{B_{n}\right\}$ be chosen so that $B \subset B_{n+1} \subset B_{n} ; B_{n}$ is measurable; and $\mu\left(B_{n}\right) \leqq \beta+1 / n$, for $n=1,2, \cdots$. Let $f_{n}(x)=\mu\left(x A \cap B_{n}\right)$. Then $f_{n} \geqq f_{n+1} \geqq 0 ; f_{n}$ is continuous; and $\int f_{n}(x) d \mu(x)=\int \mu\left(x A \cap B_{n}\right) d \mu(x)$ $=\int\left(\int \chi_{x A}(y) \chi_{B_{n}}(y) d y\right) d x=\int \chi_{B_{n}}(y)\left(\int \chi_{y A^{-1}}(x) d x\right) d y=\mu\left(A^{-1}\right) \mu\left(B_{n}\right)$ for $n=1,2, \cdots$. Now $\alpha \beta=\mu\left(A^{-1}\right) \mu^{*}(B) \leqq \mu\left(A^{-1}\right) \mu\left(B_{n}\right) \leqq \alpha(\beta+1 / n)$, for all $n$. Therefore, if we set $\bar{f}=\inf _{n} f_{n}$, then $\bar{f}$ is measurable and $\int_{G} \bar{f}(x) d \mu(x)=\alpha \beta$. It remains only to show $f(x)=\bar{f}(x)$, i.e. that

$$
\mu^{*}(x A \cap B)=\inf _{n} \mu\left(x A \cap B_{n}\right) .
$$

We know that

$$
\begin{aligned}
& \mu^{*}(B)=\mu^{*}(x A \cap B)+\mu^{*}(B \backslash x A) ; \\
& \mu\left(B_{n}\right)=\mu\left(x A \cap B_{n}\right)+\mu\left(B_{n} \backslash x A\right) ;
\end{aligned}
$$

and clearly,

$$
\mu\left(x A \cap B_{n}\right) \geqq \mu^{*}(x A \cap B) \text { and } \mu\left(B_{n} \backslash x A\right) \geqq \mu^{*}(B \backslash x A) .
$$

Therefore, since $\mu^{*}(B)=\inf _{n} \mu\left(B_{n}\right)$, it must be that $\mu^{*}(x A \cap B)$ $=\inf _{n} \mu\left(x A \cap B_{n}\right)$ and the lemma is proved.

ReMARKs. (1) Note that we have actually proved $f$ is uniformly continuous. (2) Easy examples, such as a strip in the plane, show that the assertion is false when the measure of $A$ is infinite.

Proof of Theorem 1 . There is no loss of generality in assuming $A$ and $B$ have finite outer measure. Define $f(x)=\mu^{*}\left(x A^{-1} \cap B\right)$, and let $V=\{x: f(x)>0\}$; by Lemma $1.1, V$ is a nonempty open set. If $x \in V$, then $x A^{-1} \cap B$ cannot be empty, i.e., there are elements $a, b$ 
of $A$ and $B$, respectively, such that $x a^{-1}=b ; x=b a$. This argument shows that $V \subset B A$ and concludes the proof.

We remark here that, in general, the above theorems are not true if neither $A$ nor $B$ is required to be measurable.

3. Applications. Obviously, Theorem 1 provides us with considerable information concerning semigroups in a topological group. We formulate, here, some of the applications. Unless otherwise stated, $G$ is assumed to be a locally compact topological group with invariant Haar measure $\mu$.

THEOREM 2. Let $S$ be a subsemigroup of $G$ which contains a subset of positive measure. Then the interior of $S$ is not void. In particular, (1) if $S$ is a group then $S$ is open and closed, and (2) if $G$ is $n$-dimensional Euclidean space $E^{n}$, then $S$ contains an open sector.

Proof. The first assertion is a consequence of Theorem 1 and the definition of a semigroup. If a group contains a nonempty open set, then it is open and closed which implies (1), while (2) follows from the observation that every open set in $E^{n}$ generates a semigroup containing a sector.

Proof of Theorem 3. (The statement appears in the introduction.) By Theorem 2, if $S$ is a semigroup which contains a set of positive measure, then $S^{0}$ (= the interior of $S$ ) is nonvoid. Now $S^{0}$ is an open semigroup, since the interior of a semigroup is again a semigroup, and we now employ a result of Fred B. Wright [5] which asserts that an open semigroup of a compact group is a closed subgroup. Thus, the identity $e$ of $G$ is in the interior of $S$, and it follows that $S$ is open; $S$ is an open and closed subgroup.

We have shown that for "large" $A$ and $B$ it often happens that $A B$ contains an open set. On the other hand if $G$ is a commutative compact group with an element of infinite order, it will follow from Theorem 3 that there are subsets $S$ and $T$ such that $S \cup T=G$ and neither $S^{2}$ nor $T^{2}$ contains a nonvoid open set.

To prove this last statement, we will need the following purely algebraic lemma:

Lemma 4.1. Let $H$ be an Abelian group and let $h \in H$. If $S \subset H$ is a semigroup containing $h$ but not containing $-h$, and if $S$ is maximal with respect to this property, then $S \cup-S=H$.

Proof. Suppose, to the contrary, that there is an element $t \in H$ such that $t \notin S \cup-S$. Obviously $-t \notin S \cup-S$, so by the maximality of $S$, it follows that there is some positive integer $n$ and $s_{1} \in S$ with 
$-h=s_{1}+n(-t)$. Therefore, we may now pick $n^{\prime}$ to be the smallest positive integer with the property $n^{\prime} t \in S$. Similarly, there is a positive integer $m$ and an $s_{2} \in S$ such that $-h=s_{2}+m t$. There exist positive integers $p, r$ with $0 \leqq r<n^{\prime}$ and $m=p n^{\prime}-r$; but $r t=-m t+p n^{\prime} t$ $=s_{2}+h+p n^{\prime} t \in S$; hence $r=0$. This gives $-h=s_{2}+p n^{\prime} t \in S$, a contradiction, and the proof is complete.

As for the statement preceding Lemma 4.1, assume that $G$ is a compact Abelian group having an element of infinite order. By Zorn's Lemma, the $S$ of Lemma 4.1 exists. Moreover, $S^{2} \subset S$ cannot contain an open set or $S$ would be a group, by Theorem 3 ; the same statement holds for $-S$; and $S \cup-S=G$.

We now make the further claim that $S$ is not measurable. For, if it were, then its measure must be positive and its interior would be nonempty. In particular, we have proved the following:

TheOREM 4. In a compact Abelian group, every maximal semigroup which is not a group is not Haar measurable.

Of course, such semigroups may not exist, but if the group has one element of infinite order, then it can be shown that there is a maximal semigroup which is not a group.

Our final theorem shows that the term "semigroup" is not a misnomer. Roughly, it says that if a semigroup is "more than one-half of a group," then it is indeed a group.

Theorem 5. Let $S$ and $T$ be measurable subsets of $G$ such that (1) $S$ is a semigroup, (2) $T$ is a group, and (3) $\mu(S \Delta T)<\mu(T) / 2$. Then $S=T$.

We first prove the following lemma.

Lemma 5.1. If $A$ and $B$ are measurable subsets of $G$ such that (1) $A$ is a semigroup, (2) $B$ is a group, (3) $B \subset A$, and (4) $\mu(A \backslash B)<\mu(B)$. Then $A=B$.

Proof. By way of contradiction, suppose that $x \in A \backslash B$. Then, since $A$ is a semigroup and $B$ is a group, we have $(x B) \subset A \backslash B$. But $\mu(x B)$ $=\mu(B)>\mu(A \backslash B)$, and the lemma follows.

In the theorem, first consider the case where $\mu(T)=\infty$. If $A$ is the set of $s \in S \cap T$ such that $s^{-1} \notin S \cap T$, then $A^{-1} \subset T \backslash S$. Therefore $\mu\left(S \cap S^{-1} \cap T\right)=\infty$ and $\mu\left(T \backslash\left(S \cap S^{-1}\right)\right)<\infty$. The lemma then implies that $T=S \cap S^{-1} \cap T$. We now have a group $T$ in $S$ satisfying the hypothesis of the lemma; that is $T=S$.

In case $\mu(T)<\infty$, then $T$ is compact; and $S \cap T$ is a semigroup of positive measure in $T$. So by Theorem 3 and the lemma, $S \cap T=T$; $S=T$. 


\section{REFERENCES}

1. A. B. Simon, Vanishing algebras, to appear in Trans. Amer. Math. Soc.

2. E. J. McShane, Images of sets satisfying the condition of Baire, Ann. of Math. vol. 51 (1950) pp. 380-386.

3. B. J. Pettis, On continuity and openness of homomorphisms in topological groups, Ann. of Math. vol. 52 (1950) pp. 293-308.

4. - Remarks on a theorem of E. J. McShane, Proc. Amer. Math. Soc. vol. 2 (1951) pp. 166-171.

5. Fred B. Wright, Semigroups in compact groups, Proc. Amer. Math. Soc. vol. 7 (1956) pp. 309-311.

Tulane University

\section{SIMPLE NODAL NONCOMMUTATIVE JORDAN ALGEBRAS}

\section{LOUIS A. KOKORIS}

1. Introduction. Nodal algebras were defined by R. D. Schafer [4] and have also been studied by the author $[2 ; 3]$. A noncommutative Jordan algebra is an algebra $\mathfrak{A}$ over a field $\mathfrak{F}$ satisfying (1) the flexible law $(x y) x=x(y x)$ and (2) the condition that $\mathfrak{A}^{+}$is a Jordan algebra. That is, $\mathfrak{A}^{+}$satisfies the identity $\left(x^{2} \cdot y\right) \cdot x=x^{2} \cdot(y \cdot x)$ where we have used the dot to indicate the product of $\mathfrak{A}^{+}$. The algebra $\mathfrak{A}^{+}$ is defined to be the same vector space as $\mathfrak{A}$ but with product $x \cdot y$ $=(x y+y x) / 2$ where $x y$ and $y x$ are products in $\mathfrak{A}$. Then $\mathfrak{A}$ is called nodal if it is finite dimensional, if $\mathfrak{A}$ has identity element 1 , if $\mathfrak{A}$ can be written as a vector space direct sum $\mathfrak{A}=\mathfrak{F} 1+\mathfrak{N}$ where $\mathfrak{N}$ is the subspace of nilpotent elements of $\mathfrak{A}$, and if $\mathfrak{N}$ is not a subalgebra of $\mathfrak{A}$.

Every known nodal algebra $\mathfrak{A}$ has the property that $\mathfrak{U}^{+}$is an associative algebra. The flexible algebras with $\mathfrak{A}^{+}$associative have been described in [3]. In this paper we shall prove the following theorem.

TheоReм 1. Let $\mathfrak{i}$ be a simple nodal noncommutative Jordan algebra of characteristic $\neq 2$. Then $\mathfrak{A}^{+}$is associative.

Define $\mathfrak{B}$ to be the subspace of $\mathfrak{A}$ generated by all the associators in $\mathfrak{N}^{+}$. That is, $\mathfrak{B}$ is generated by elements of the form $(x \cdot y) \cdot z-x \cdot(y \cdot z)$ with $x, y, z$ in $\mathfrak{R}$. The proof of the theorem will be made by showing that the ideal $\mathfrak{S}$ of $\mathfrak{A}$ generated by $\mathfrak{B}$ is not all of $\mathfrak{A}$ and since $\mathfrak{A}$ is simple it will follow that $\mathfrak{C}=0$ and $\mathfrak{B}=0$. This is the desired result.

The original proof was not valid when the characteristic is 3 . The author thanks Professor R. D. Schafer for suggesting a modification

Presented to the Society, January 28, 1958; received by the editors January 13 , 1958. 